\title{
Performance Analysis of Flameproof Submersible Pump for Mining Application
}

\author{
Ayuba Elkanah Jatau', Israel Enock Ojo ${ }^{2}$, Elvis Adam Alhassan ${ }^{3}$, Israel Enema Ohiemi ${ }^{4, *}$ Kolo Yetu \\ Babazhitsu $^{2}$ \\ ${ }^{1}$ Department of Mechanical Engineering Technology, Niger State Polytechnic, Zungeru, Nigeria. \\ ${ }^{2}$ Department of Mechanical Engineering, Federal Polytechnic, Bida, Niger State, Nigeria. \\ ${ }^{3}$ Faculty of Mathematical Sciences, C. K. Tedam University of Technology and Applied Sciences, Navrongo, Upper East Region, \\ Ghana. \\ ${ }^{4}$ Department of Mechanical Engineering, University of Nigeria, Nsukka, Nigeria.
}

How to cite this paper: Ayuba Elkanah Jatau, Israel Enock Ojo, Elvis Adam Alhassan, Israel Enema Ohiemi, Kolo Yetu Babazhitsu. (2021) Performance Analysis of Flameproof Submersible Pump for Mining Application. Engineering Advances, $1(2)$, 39-46.

DOI: 10.26855/ea.2021.12.002

Received: November 1, 2021

Accepted: November 26, 2021

Published: December 21, 2021

*Corresponding author: Israel Enema Ohiemi, Department of Mechanical Engineering, University of Nigeria, Nsukka, Nigeria.

Email: israel.ohiemi@unn.edu.ng

\begin{abstract}
This paper seeks to predict and compare the performance of flameproof pumps same design parameters but with different operation stages. Firstly, two pump cases A and B were modelled. Secondly, the shear stress transport (SST) turbulence model was used to solve the 3D Navier Stokes equation. The performance characteristics were obtained and compared with experimental data. The pressure contours are plotted at the design point to delineate its internal flow characteristics. Pump case A was found to require more power for its operation and would hence consume more energy than pump case B. Further analysis shows that pump $\mathrm{B}$ is more efficient in operation, but with complex configuration. Lastly, the numerical simulations were validated with experimental data.
\end{abstract}

\section{Keywords}

Flameproof Submersible Pump, Performance, Simulation, Fluid-Structure Interaction, Optimization

\section{Introduction}

Pumps used on mine site are flameproof, submersible, light weight, low noise, stable in operation, multi-stage and operates with a head capacity of 450 meters [1]. The high head requirement is significant so as to discharge the sewage within the mine to the surface without a need for additional pumps nor reservoirs along the flow path [2].

Africa is blessed with large deposits of mineral resources but for her to attain a robust economic growth, special attention must be given to these natural resources. These large mineral resources when explored would help to drive industrial growth. Mining pumps are necessary on mine sites since they are needed to move fluid from low depth to a higher surface [3]. To effectively improve mining operations, the performance of mining pump must be addressed.

In recent times, several scholars presented numerical and experimental studies on high-speed pumps. Draghici et al. [4] outlined experimental test on centrifugal pump at different speeds. Georgescu et al. [5] examined the characteristic pump curves with two speeds based on EPANET. Rakibuzzaman et al. [6] examined performance of a multistage pump characteristics using inverter controlled speed variable. Sha et al. [7] obtained the internal with centrifugal flow pattern at variable speeds both numerically and via experiments.

Furthermore, the use of computational fluid dynamics (CFD) to optimize and design pump has become a useful analytical tool in evaluating the flow characteristics inside the pump. This method can also be used to obtain fluid flow performance within the pump's flow channels. A number of researchers explored the merits of CFD in pump performance analysis [8-11].

The increasing demand for draining pump used on mine is attributed to the increase and the expansion of mining sites [12]. The flameproof submersible pumps (FSP) and its motor are closely integrated into one unit and commonly drive 
operation. FSP should function freely with low noise. However, FSP are failure prone as a result of its design and the harsh working conditions. The average operation time before first failure of FSP is approximately 3,800 hours, hence detrimental to its performance [8].

In view of the above challenges, a thorough assessment of this class of pump is essential. Pump BQS50-450/2-200 and BQS50-450/6-160 referred to as Case A and Case B were selected for this study. The case studies were modelled, simulated and their results verified experimentally.

\section{Theoretical Framework}

\subsection{Governing Equations}

The flow interaction within the flame proof pumps was studied by means of numerical computations. The Numerical calculations were executed based on the continuity and Reynolds averaged Navier-Stokes equations for incompressible as presented in equations 2 and 3.

$$
\begin{gathered}
\frac{d u_{i}}{d x_{i}}=0 \\
\rho\left[\frac{\partial \overline{u_{i}}}{\partial t}+\frac{\partial\left(\overline{u_{j} u_{i}}\right)}{\partial x_{j}}\right]=\frac{\partial}{\partial x_{j}}\left[\mu\left(\frac{\partial \overline{u_{i}}}{\partial x_{j}}\right)-\rho \overline{u_{j}^{\prime} u_{i}^{\prime}}\right]-\frac{\partial \bar{p}}{\partial x_{i}}
\end{gathered}
$$

\subsection{Turbulence Model}

Due to the intricate nature of the pumps under investigation, a suitable turbulence model (shear stress transport SST) with a unique feature of blending the transformed $k-\omega$ and $k-\varepsilon$ equations was selected. SST automatically alternates between both models to address flow problems both along the wall or free regions. Menter [13] and Wilcox [14] proposed SST as an enhancement of $k-\omega$ turbulence model. Menter [13] established the function used by Wilcox [14] to enhance SST's sensitivity to free flow streams. Menter [13], further modified $k-\omega$ model by the introducing F1 and (1-F1) in $k-\varepsilon$ equation. the suitability of SST in resolving turbulence in pumps is well documented [15-17]

$k-\omega$ equations:

$$
\begin{gathered}
\frac{\partial(\rho k)}{\partial t}+\frac{\partial}{\partial x_{j}}\left(\rho U_{j} k\right)=\frac{\partial}{\partial x_{j}}\left[\left(\mu+\frac{\mu_{t}}{\sigma_{k 1}}\right) \frac{\partial k}{\partial x_{j}}\right]+P_{k}-\beta^{\prime} \rho k \omega \\
\frac{\partial(\rho \omega)}{\partial t}+\frac{\partial}{\partial x_{j}}\left(\rho U_{j} \omega\right)=\frac{\partial}{\partial x_{j}}\left[\left(\mu+\frac{\mu_{t}}{\sigma_{\omega 1}}\right) \frac{\partial \omega}{\partial x_{j}}\right]+\alpha_{1} \frac{\omega}{k} P_{k}-\beta_{1} \rho \omega^{2}
\end{gathered}
$$

Tranformed $k-\varepsilon$ model :

$$
\begin{gathered}
\frac{\partial(\rho k)}{\partial t}+\frac{\partial}{\partial x_{j}}\left(\rho U_{j} \mathrm{k}\right)=\frac{\partial}{\partial x_{j}}\left[\left(\mu+\frac{\mu_{t}}{\sigma_{k 2}}\right) \frac{\partial k}{\partial x_{j}}\right]+P_{k}-\beta^{\prime} \rho k \omega \\
\frac{\partial(\rho \omega)}{\partial t}+\frac{\partial}{\partial x_{j}}\left(\rho U_{j} \omega\right)=\frac{\partial}{\partial x_{j}}\left[\left(\mu+\frac{\mu_{t}}{\sigma_{\omega 2}}\right) \frac{\partial \omega}{\partial x_{j}}\right]+2\left(1-F_{1}\right) \rho \frac{1}{\sigma_{\omega 2} \omega} \frac{\partial k}{\partial x_{j}} \frac{\partial \omega}{\partial x_{j}}+\alpha_{2} \frac{\omega}{k} P_{k}-\beta_{2} \rho \omega^{2}
\end{gathered}
$$

The blending functions $F_{1}$ and $F_{2}$ are:

$$
\begin{aligned}
& F_{1}=\tanh \left(\Gamma_{1}^{4}\right) \\
& F_{2}=\tanh \left(\Gamma_{2}^{2}\right)
\end{aligned}
$$

The turbulent viscosity, $\mu_{t}$ is calculated using: 


$$
\mu_{t}=\frac{\alpha_{1} k \rho}{\max \left(\alpha_{1} \omega, S F_{2}\right)}
$$

The $k-\omega$ equation for the inner boundary layer prediction is applied using the following coefficients: $\sigma_{k 1}=1.176, \sigma_{\omega l}$ $=2.0, \beta 1=0.075, \alpha_{1}=5 / 9, \beta^{\prime}=0.09$ [18]. Meanwhile, the $k-\varepsilon$ equation for the free-stream flows is solved using the following coefficients: $\sigma_{k 2}=1.0, \sigma_{\omega 2}=1 / 0.856, \beta_{2}=0.828$.

\subsection{Pump Performance Evaluation}

To effectively establish performance for pumps, estimating its overall head as presented in equation (11) is a fundamental requirement [19].

$$
H=\frac{P_{1}-P_{2}}{\rho g}
$$

where $P(m)$ denotes the pressure head, $\rho\left(\mathrm{kg} / \mathrm{m}^{3}\right)$ denote fluid density, $g\left(\mathrm{~m} / \mathrm{s}^{2}\right)$ denote the gravitational acceleration subscript 1 and 2 denote inlet and outlet of pump. Efficiency as outlined in equation (12) is another important variable for determining pump performance.

$$
\eta=\frac{\rho g Q H}{P}
$$

where the $P$ is the power input and is given as:

$$
P=T \omega
$$

\section{Methodology}

\subsection{Computational Fluid Dynamics}

The theoretical equations presented in Section 2 were solved using computational fluid dynamics (CFD). CFD steps are outlined in Figure 1.

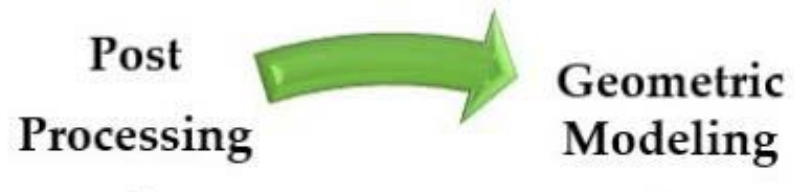

5

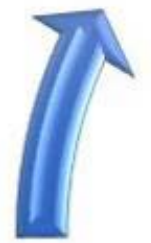

Solving

4
1

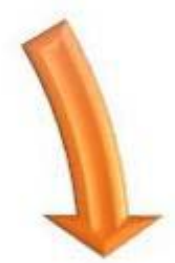

Meshing

2

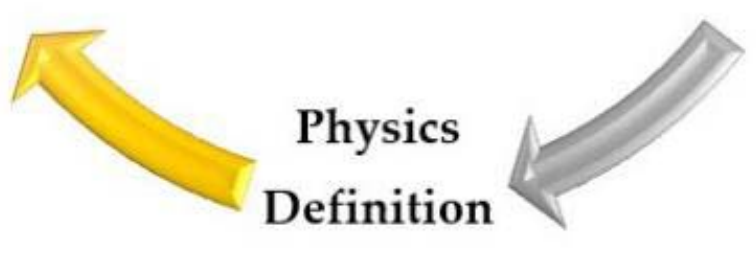

Figure 1. Steps involved in Numerical Simulations. 


\subsection{Pump Model}

Two in-built fireproof multi-stage submersible pump BQS50-450/2-200 and BQS50-450/6-160 which would be classified as pump case A and case B respectively are presented in Figure 2 and Table 1. Pump case A and B share similar design specification with head of $450 \mathrm{~m}$, flowrate of $13.9 \mathrm{l} / \mathrm{s}$ and rotation speed 2,950. The fluid path of pump cases A and B were modelled using CREO Parametric 7.0.

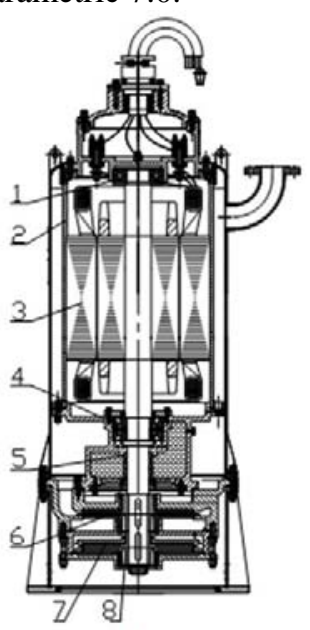

(a)

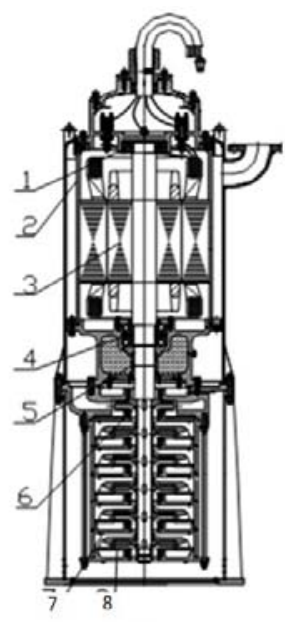

(b)

1. Deep groove ball bearings; 2. Housing; 3. Motor rotor; 4. Angular contact ball bearings;

5. Mechanical seal; 6. Final stage impeller; 7. Guide vane; 8. Head impeller.

Figure 2. Pump Models: (a) Case A; and (b) Case B.

Table 1. Main geometric parameters of the pump

\begin{tabular}{cccccccc}
\hline Case & $D_{\mathrm{j}}(\mathrm{mm})$ & $D_{2}(\mathrm{~mm})$ & $b_{2}(\mathrm{~mm})$ & $\beta_{2}\left({ }^{\circ}\right)$ & $\varphi_{\left({ }^{\circ}\right)}$ & $z$ & $D_{3}(\mathrm{~mm})$ \\
\hline A & 115 & 393 & 14 & 11 & 132 & 7 & 410 \\
B & 105 & 249 & 22 & 9 & 173 & 4 & 270 \\
\hline
\end{tabular}

\subsection{Mesh Generation}

The meshing of the fluid path was executed with ANSYS ICEM by means of hexahedral structural grid using multi-blocks. The intricate pump sections were refined with more grids so as to ensure numerical accuracy. Grid independent test was conducted in line with Celik et al. [18] grid C with 1883755 was selected using pump case A. Pump case B was grided with same technique. See Figures 3 and 4 and Table 2 for detailed grid report for each pump component.
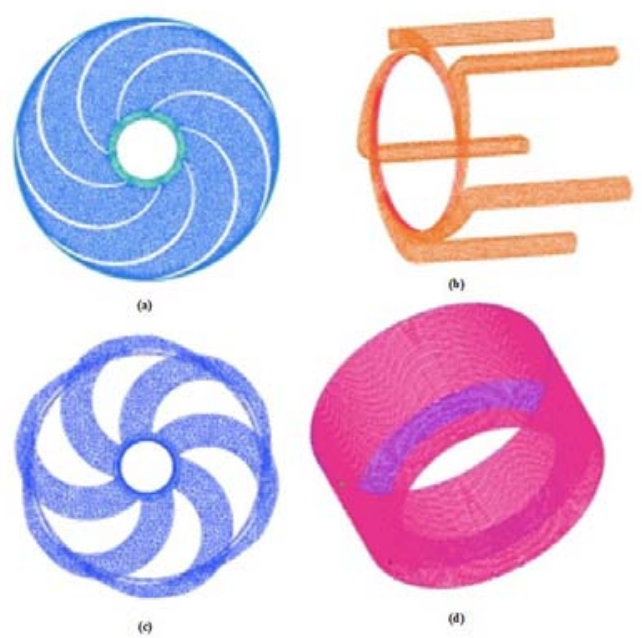

Figure 3. Computational Mesh for Pump Case A: (a) impeller (b) volute (c) guide vane w (d) mouth ring gap/cavity. 


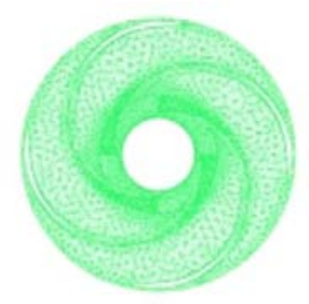

(a)

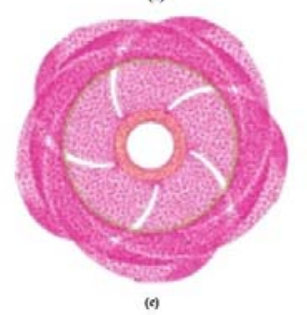

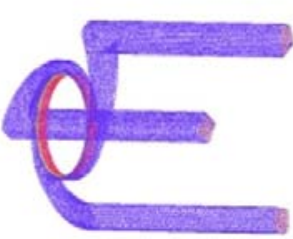

(b)

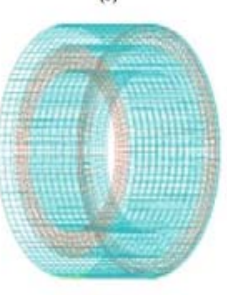

(d)

Figure 4. Computational Mesh for Pump Case B: (a) impeller (b) volute (c) guide vane w (d) mouth ring gap/cavity.

Table 2. Number of main water grids for pumps

\begin{tabular}{lllllll}
\hline Model & Impeller & Guide vane & Volute & Gaps/cavities & Others & total \\
\hline Case A & 959,768 & 152,564 & 113,019 & 193,769 & 706,084 & $1,975,291$ \\
Case B & 539,038 & 958,390 & 90,980 & 17,192 & 436,191 & $1,879,272$ \\
\hline
\end{tabular}

\subsection{Physics Definition}

CFX a sub-program under ANSYS was employed to evaluate the flow equations inside the pump. The computational set up was defined as shown in Table 3.

Table 3. Computational Physics Definition

\begin{tabular}{lll}
\hline Domain Location & Boundary Type & Mass and Momentum \\
\hline Inlet Pipe & Inlet & Total Pressure \\
Outlet pipe & Outlet & Opening \\
Domain Surface & Wall & \\
& & \\
Turbulence Model & Standard SST k- $\omega$ & \\
Model Applied & & \\
& & \\
Interface Configuration & Frozen rotor & \\
Steady State & & \\
Numerical Calculation Solver Control & 1000 & $1.15 \times 10-4 \mathrm{~s}$ \\
No of iterations & $10^{-5}$ \\
Timestep & General Grid Interface (GGI) \\
RMS residual target & \\
Mesh connection &
\end{tabular}

\section{Result and Discussion}

\subsection{Performance Characteristics Curve}

External characteristics of the pump cases A and B were analysed and compared using equations 11 and 12 . These 
characteristics comprehensively and intuitively express the pump performance in form of curves shown in Figure 5 . The complex nature of flow in pump is unsteady and requires experiments to authenticate pump performance. As a result, CFD results for pump cases A and B was compared with experimental data provided by pump manufacturer in Figure 5. It was observed from Figure 5 as the flow rises, head decreases but shaft power increases. The data evaluated from CFD is higher than experimental data. The experimental process is prone to friction and volume losses which are not considered in CFD.

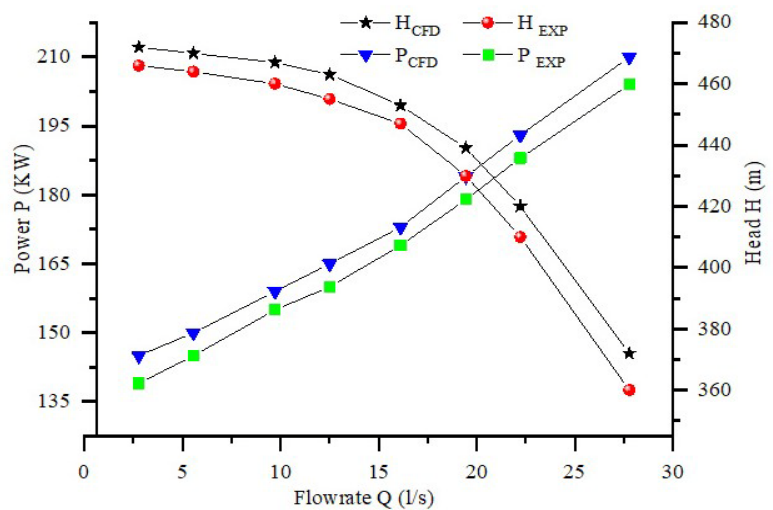

(a)

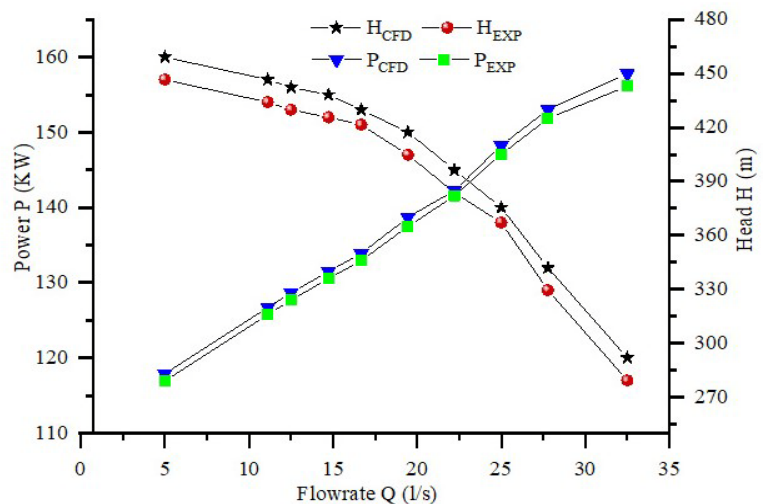

(b)

Figure 5. Performance Curves Pump Cases: (a) case A (b) case B.

The result depicts that both pumps exhibit similar performance trend. However, Pump case A performs at a higher head than Case B. Therefore, case A is endorsed for high head. On the contrary, the power requirement for pump case B is low, hence more energy would be saved using Pump case B. However, its structural make up is complex to either build or maintain.

\subsection{Internal Flow Characteristics}

Figure 6 and 7, depicts pressure contours at design flow. It was observed that the lowest pressure was recorded at the inlet region for both pump cases. Gradual upward trend in pressure within pump’s flow channel was observed as maximum value was registered at pump outlet. The fluid pressure rises gradually and is equally distributed along the flow circumference. The impeller flow passage, as presented in Figures 6 and 7, accounts for low pressure at the mid-section when viewed radially outward. The impeller's pressure (see Figure 7) increases radially from its mid-section to its maximum value at its edges. The distribution in pressure at the design condition is circumferentially symmetry as presented in Figure 6 and Figure 7. A low-pressure zone was observed at the inlet which means that this zone is prone to cavitation.

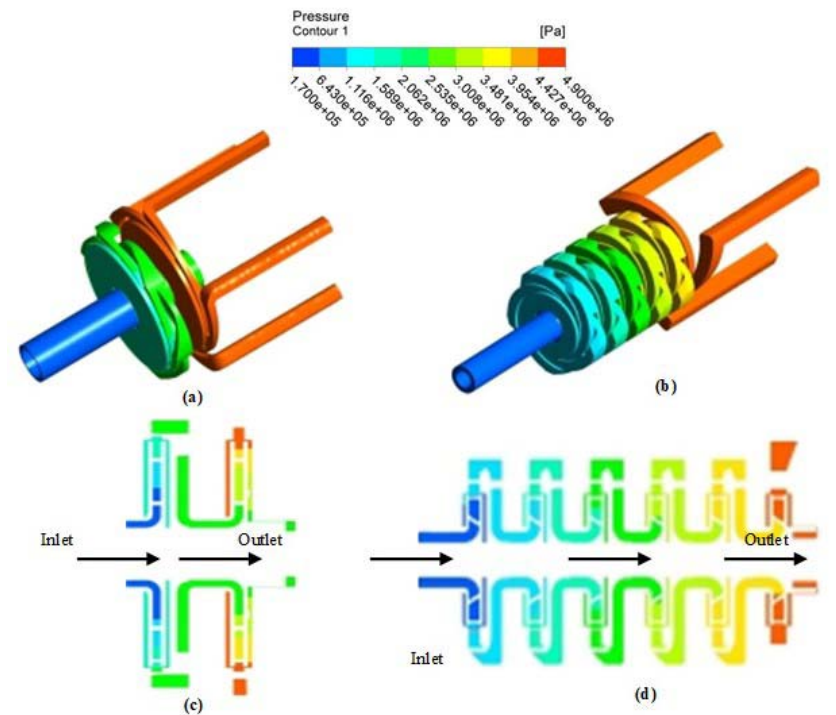

Figure 6. Pressure Contour plots: (a) 3D of case A (b) $3 D$ of case $B$ (c) Case A at plane $x=0$ (d) Case $B$ at plane $x=0$. 


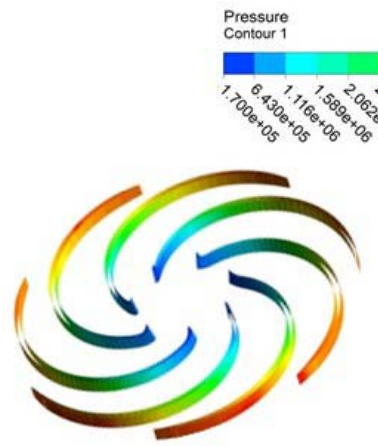

(a)

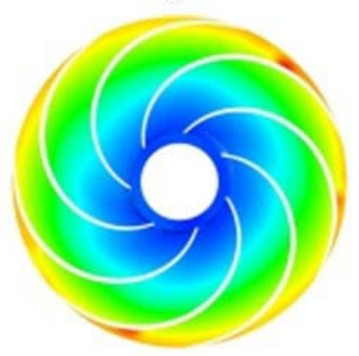

(c)
$[\mathrm{Pa}]$

(Pal

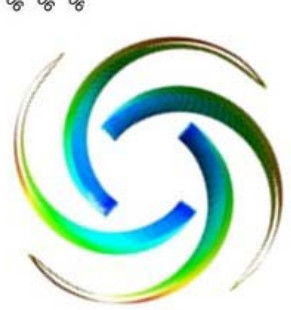

(b)

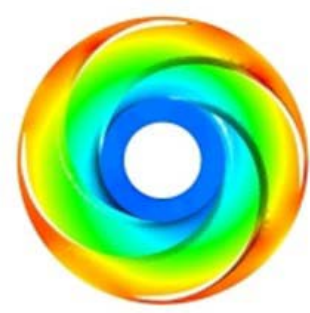

(d)

Figure 7. Pressure Contour plots: (a) blades of Case A (b) blades of case B (c) Impeller of Case A (d) Impeller of Case B.

\section{Conclusion}

Two pump models for mining operation were modelled using CREO parametric software. Their fluid path was meshed using ANSYS ICEM CFD. The pump cases were separately imported into ANSYS CFX and the Shear Stress Transport turbulent model was used to solve the continuity and momentum equation in the flow channels of the pump. After post processing, the performance of both pumps were analysed and compared with experimental values. The pressure distribution and external characteristics of the two pumps which shows an increasing trend of pressure from the pump inlet to outlet was observed in both pump cases. The analysis shows that pump B is more efficient in operation, but the structure is more complex as compared with pump A. Furthermore, the design, manufacturing and cost implication of pump case B is relatively high.

\section{References}

[1] J. F. Gülich. (2014). Centrifugal Pumps, Springer Berlin Heidelberg, Berlin, Heidelberg, 2014. https://doi.org/10.1007/ 978-3-642-40114-5.

[2] Y. Wu, X. Xiao, Z. Song. (2017). Competitiveness analysis of coal industry in China: A diamond model study. Resour. Policy. 52 (2017). https://doi.org/10.1016/j.resourpol.2017.01.015.

[3] J. Liu, T. Yang, L. Wang, X. Chen. (2020). Research progress in coal and gas comining modes in China . Energy Sci. Eng., 8 (2020). https://doi.org/10.1002/ese3.739.

[4] I. Drăghici, C. Atănăsoaiei, A. Bosioc, S. Muntean, L. E. Anton. (2017). Experimental Analysis of the Global Performances for a Pump with Symmetrical Suction Elbow at Two Speeds. Energy Procedia, 112(2017), 225-231. https://doi.org/10.1016/j.egypro.2017.03.1090.

[5] A.-M. Georgescu, C.-I. Cosoiu, S. Perju, S.-C. Georgescu, L. Hasegan, A. Anton. (2014). Estimation of the Efficiency for Variable Speed Pumps in EPANET Compared with Experimental Data. Procedia Eng., 89(2014), 1404-1411. https://doi.org/10.1016/j.proeng.2014.11.466.

[6] Rakibuzzaman, S.-H. Suh, K., Kyung-Wuk, H.-H. Kim, M.T. Cho, I.S. Yoon. (2015). A Study on Multistage Centrifugal Pump Performance Characteristics for Variable Speed Drive System. Procedia Eng., 105(2015), 270-275. https://doi.org/10.1016/j.proeng.2015.05.109.

[7] Y. Bai, F. Kong, F. Zhao, J. Wang, B. Xia, Q. Hu. (2018). Experiments and Numerical Simulation of Performances and Internal Flow for High-Speed Rescue Pump with Variable Speeds. Math. Probl. Eng., 2018(2018), 1-18. https://doi.org/10.1155/2018/9168694. 
[8] H. Pineda, J. Biazussi, F. López, B. Oliveira, R. D. M. Carvalho, A. C. Bannwart, N. Ratkovich. (2016). Phase distribution analysis in an Electrical Submersible Pump (ESP) inlet handling water-air two-phase flow using Computational Fluid Dynamics (CFD). J. Pet. Sci. Eng., 139(2016), 49-61. https://doi.org/10.1016/j.petrol.2015.12.013.

[9] M. Zhang, D. Valentin, C. Valero, A. Presas, M. Egusquiza, E. Egusquiza. (2020). Experimental and numerical investigation on the influence of a large crack on the modal behaviour of a Kaplan turbine blade. Eng. Fail. Anal., 109(2020). https://doi.org/10.1016/j.engfailanal.2020.104389.

[10] N. Zhang, M. Yang, B. Gao, Z. Li, D. Ni. (2015). Experimental and numerical analysis of unsteady pressure pulsation in a centrifugal pump with slope volute. J. Mech. Sci. Technol., 29(2015), 4231-4238. https://doi.org/10.1007/s12206-015-0919-y.

[11] H. Jian, W. Kaiqiang, W. Chao, G. Lang, G. Chunyu. (2021). Prediction of hydrodynamic performance of pump jet propulsor considering the effect of gap flow model. Ocean Eng., 233(2021), 109162. https://doi.org/10.1016/j.oceaneng.2021.109162.

[12] J. Wang, Z. Huang. (2017). The Recent Technological Development of Intelligent Mining in China. Engineering, 3(2017), 439-444. https://doi.org/10.1016/J.ENG.2017.04.003.

[13] F. Menter. (1993). Zonal Two Equation k-w Turbulence Models For Aerodynamic Flows, in: 23rd Fluid Dyn. Plasmadynamics, Lasers Conf., American Institute of Aeronautics and Astronautics, Reston, Virigina, 1993. https://doi.org/10.2514/6.1993-2906.

[14] D. C. Wilcox. (1994). Simulation of Transition with a Two-Equation Turbulence Model, AIAA J. 32 (1994). https://doi.org/10.2514/3.59994.

[15] M. Böhle, A. Fleder, M. Mohr. (2019). Study of the losses in fluid machinery with the help of entropy. Open Arch. 16th Int. Symp. Transp. Phenom. Dyn. Rotating Mach. ISROMAC 2016. (2019).

[16] M. H. Sotoude Haghighi, S. M. Mirghavami, S. F. Chini, A. Riasi. (2019). Developing a method to design and simulation of a very low head axial turbine with adjustable rotor blades. Renew. Energy., 135(2019), 266-276. https://doi.org/10.1016/j. renene.2018.12.024.

[17] M. Hadi, S. Haghighi, S. M. Mirghavami, M. M. Ghorani, A. Riasi, S. F. Chini. (2020). A numerical study on the performance of a superhydrophobic coated very low head (VLH) axial hydraulic turbine using entropy generation method. Renew. Energy., 147(2020), 409-422. https://doi.org/10.1016/j.renene.2019.09.003.

[18] I. B. Celik, et al. (2008). Procedure for Estimation and Reporting of Uncertainty Due to Discretization in CFD Applications. J. Fluids Eng., 130(2008), 078001. https://doi.org/10.1115/1.2960953.

[19] J. Pei, F. Zhang, D. Appiah, B. Hu, S. Yuan, K. Chen, S. N. Asomani. (2019). Performance prediction based on effects of wrapping angle of a side channel pump. Energies, 12(2019). https://doi.org/10.3390/en12010139. 